\title{
Heteromultimeric Potassium Channels Formed by Members of the Kv2 Subfamily
}

\author{
Judith T. Blaine and Angeles B. Ribera \\ Department of Physiology and Biophysics, University of Colorado Health Sciences Center, Denver, Colorado 80262
}

Four $\alpha$-subunits are thought to coassemble and form a voltagedependent potassium $\left(\mathrm{K}_{\mathrm{v}}\right)$ channel. $\mathrm{K}_{\mathrm{v}} \alpha$-subunits belong to one of four major subfamilies (Kv1, Kv2, Kv3, Kv4). Within a subfamily up to eight different genetic isotypes exist (e.g., Kv1.1, Kv1.2). Different isotypes within the Kv1 or Kv3 subfamily coassemble. It is not known, however, whether the only two members of the vertebrate Kv2 subfamily identified thus far, Kv2.1 and Kv2.2, heteromultimerize. This might account for the lack of detection of heteromultimeric Kv2 channels in situ despite the coexpression of Kv2.1 and Kv2.2 mRNAs within the same cell. To probe whether Kv2 isotypes can form heteromul- timers, we developed a dominant-negative mutant Kv2.2 subunit to act as a molecular poison of Kv2 subunit-containing channels. The dominant-negative Kv2.2 suppresses formation of functional channels when it is coexpressed in oocytes with either wild-type Kv2.2 or Kv2.1 subunits. These results indicate that Kv2.1 and Kv2.2 subunits are capable of heteromultimerization. Thus, in native cells either Kv2.1 and Kv2.2 subunits are targeted at an early stage to different biosynthetic compartments or heteromultimerization otherwise is inhibited.

Key words: potassium channels; heteromultimers; coassembly; Kv2; dominant-negative mutant; Xenopus
Voltage-dependent potassium $\left(\mathrm{K}_{\mathrm{v}}\right)$ channels play many crucial roles in neuronal function. Functional $\mathrm{K}_{\mathrm{v}}$ channels are believed to contain four pore-forming $\alpha$-subunits (MacKinnon, 1991). The genes encoding $\mathrm{K}_{\mathrm{v}} \alpha$-subunits are classified into four major subfamilies: Kv1, Kv2, Kv3, and Kv4 (Chandy, 1991). Each subfamily contains different genetic isotypes that may encode functionally distinct channels, augmenting potassium channel diversity.

Potassium channel heterogeneity is increased further by the possibility of forming homo- as well as heteromultimeric channels. Isotypes within the same subfamily form heterotetramers, indicating a barrier to subunit coassembly across subfamilies (Covarrubias et al., 1991). However, in a few cases, subunits encoded by members of different subfamilies appear to coassemble (Shahidullah et al., 1995a,b; Chen et al., 1996; Hugnot et al., 1996; Post et al., 1996; Patel et al., 1997; Salinas et al., 1997a,b). Heteromultimerization of either Kv1 or Kv3 subfamily members has been demonstrated directly in the oocyte (Christie et al., 1990; Isacoff et al., 1990; Ruppersberg et al., 1990; Weiser et al., 1994), and Kv1 subfamily heteromultimers have been detected immunohistochemically in situ (Sheng et al., 1993; Wang et al., 1993; Veh et al., 1995). Furthermore, transcripts encoded by different members of either the Kv2 or Kv4 subfamilies have been detected within the same neurons (Hwang et al., 1992; Song et al., 1998), allowing for possible heteromultimer formation. However, immunocytochemical studies indicate that Kv2.1 and Kv2.2 sub-

\footnotetext{
Received June 29, 1998; revised Sept. 3, 1998; accepted Sept. 10, 1998.

This work was supported by National Institutes of Health Grant NIH 5T32NS07083, National Research Service Award Fellowship 5 F30 MH11349-03 to J.T.B., and National Institutes of Health Grant RO1-NS25217 to A.B.R. We thank Drs. R. Joho and R. MacKinnon for their suggestions regarding mutagenesis, Dr. J. Karpen for assistance with statistical analysis, and an anonymous reviewer for insightful comments on this manuscript. We also thank H. Chouinard, A. Hofmann, Dr. M. Lazaroff, A. Linares, Dr. T. Nick, Dr. R. Scannevin, and Dr. J. Trimmer for helpful discussions.

Correspondence should be addressed to Judith T. Blaine, Department of Physiology and Biophysics, C-240, University of Colorado Health Sciences Center, Denver, CO 80262.

Copyright (C) 1998 Society for Neuroscience $\quad 0270-6474 / 98 / 189585-09 \$ 05.00 / 0$
}

units do not colocalize, although in certain neurons their encoded proteins are detectable within the same cell (Hwang et al., 1993).

Because immunocytochemical data raise the possibility that Kv2 subfamily members do not heteromultimerize, this study investigates directly whether Kv2.1 and Kv2.2 subunits can heteromultimerize with each other. When coexpressed in the oocyte, wild-type Kv2.1 and Kv2.2 subunits induce currents that are functionally very similar (Burger and Ribera, 1996) (Fig. 1). Thus, heteromultimerization would not be revealed by unique functional properties of a resultant channel, a strategy used successfully to detect Kv1 and Kv3 heteromultimers (Christie et al., 1990; Isacoff et al., 1990; Ruppersberg et al., 1990; Weiser et al., 1994). To probe whether Kv2.1 and Kv2.2 subunits heteromultimerize, we developed a mutant Kv2.2 subunit that serves as a molecular poison of Kv2.2 channels. Coexpression of mutant and wild-type Kv2.2 subunits leads to a reduction in the currents induced, indicating that the inclusion of the mutant subunit in a Kv2.2 tetramer eliminates function. Further, coexpression of dominant-negative Kv2.2 and wild-type Kv2.1 subunits also decreases functional Kv2 channel expression. These results indicate that the mutant Kv2.2 subunit poisons Kv2.1- as well as Kv2.2containing channels, providing evidence that Kv2.1 and Kv2.2 subunits are capable of heteromultimerization. Thus, the failure to detect Kv2.1/Kv2.2 heteromultimers in situ suggests the existence of either modifications of native Kv2.1 and Kv2.2 subunits that prevent their coassembly or neuronal sorting mechanisms that target each subunit to distinct biosynthetic compartments.

\section{MATERIALS AND METHODS}

RNA synthesis. The entire coding region of the Xenopus Kv2.1 gene surrounded by the $5^{\prime}$ and $3^{\prime}$ untranslated sequences of Xenopus $\beta$-globin [which confer stability and allow efficient translation of in vitro transcribed mRNAs (Liman et al., 1992)] was excised from the pGEMHEX9 construct (Burger and Ribera, 1996) and cloned into the pALTER vector (Promega, Madison, WI), creating the recombinant pA2.1 clone. The entire coding region of the Xenopus Kv2.2 gene flanked by the $5^{\prime} \beta$-globin untranslated region (UTR) likewise was removed from the pGEMHEX12-2 construct (Burger and Ribera, 1996) and inserted into 
Figure 1. Xenopus Kv2.1 and Kv2.2 transcripts induce the expression of functionally similar currents. $A$, Oocytes were injected with $0.2 \mathrm{ng}$ of wild-type Kv2.1 RNA (left), 1.8 ng of wild-type Kv2.2 RNA (center), or $0.2 \mathrm{ng}$ of Kv2.1 RNA plus $1.8 \mathrm{ng}$ of Kv2.2 RNA (right). The injection of a mixture of wild-type Kv2.1 and Kv2.2 transcripts induces larger currents than either Kv2.1 or Kv2.2 RNA injected alone. Currents were generated in response to $160 \mathrm{msec}$ voltage steps to potentials ranging from -50 to $+100 \mathrm{mV}$ from a holding potential of -80 $\mathrm{mV}$; leak-subtracted currents are shown (see Materials and Methods). Calibration: $1.9 \mu \mathrm{A}, 55 \mathrm{msec} . B$, The voltage-dependent properties of activation for the currents induced by the expression of wild-type Kv2.1 alone $(n=39)$ or a functionally equal amount of wild-type Kv2.1 and Kv2.2 RNA $(n=34)$ are shown on the left. Similar graphs for wild-type Kv2.2 currents $(n=31)$ and those induced by a functionally equal amount of wild-type Kv2.1 and Kv2.2 transcripts are shown on the right. Symbols are mean values; error bars indicate SD.

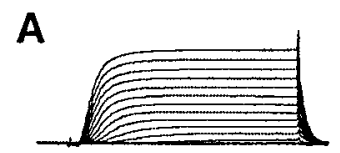

$\mathrm{Kv} 2.1$

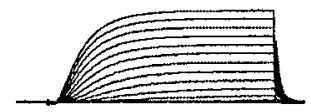

$\mathrm{Kv} 2.2$

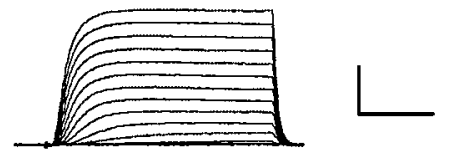

$\mathrm{Kv} 2.1+\mathrm{Kv} 2.2$

B
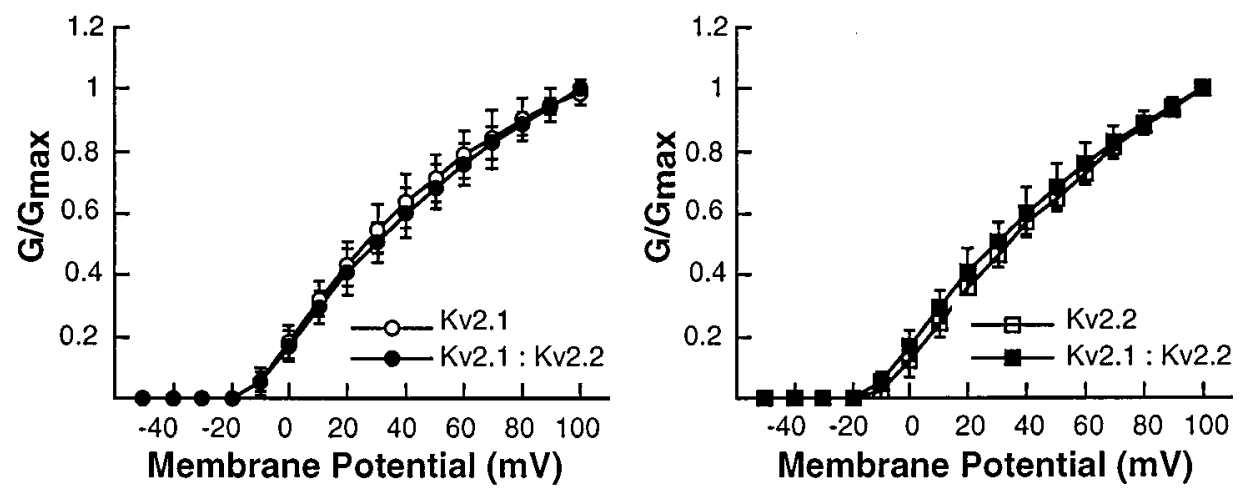

the pALTER vector to generate the recombinant pA2.2 clone. Although pGEMHEX12-2 and pA2.2 lack the 3' $\beta$-globin UTR, high expression levels are still obtained from these clones (Burger and Ribera, 1996; this study). pA2.1 and pA2.2 both were linearized with SphI. Capped, sense RNA was transcribed in vitro by using T7 RNA polymerase in the presence of ribonucleotide triphosphates (Pharmacia, Piscataway, NJ) and cap analog (Boehringer Mannheim, Indianapolis, IN). RNA concentrations and integrity were determined spectrophotometrically and by electrophoresis in agarose-formaldehyde gels in combination with ethidium bromide staining.

Site-directed mutagenesis. The tryptophan-to-phenylalanine $(\mathrm{W} \Delta \mathrm{F})$ substitution in the pore region was achieved by using the pALTER in vitro mutagenesis system according to the manufacturer's instructions (Promega). The tryptophan-to-cysteine (WDC) and tyrosine-tothreonine ( $\mathrm{Y} \Delta \mathrm{T}$ ) mutations were made with PCR cassette mutagenesis in a procedure modified from Hollmann et al. (1994), as follows. The generation of each mutation involved the use of four primers in two sequential PCR reactions. Two of the primers (Universal Forward, Universal Reverse) flank the pore region. Each contains a unique native restriction site (SalI or NcoI) used for the subsequent replacement of a PCR-generated pore cassette into the Kv2.2 gene. The two other primers (Pore Sense, Pore Antisense) recognize a sequence within the pore, are complimentary to each other, and encode the desired mutation (W $\Delta \mathrm{C}$ or $\mathrm{Y} \Delta \mathrm{T})$ as well as an engineered silent restriction site to provide an initial means of screening resultant clones. The first round of PCR involved two separate reactions, using as primers either (1) Universal Forward and Pore Antisense or (2) Universal Reverse and Pore Sense. Depending on the mutant to be generated, the template in these reactions was either wild-type Kv2.2 DNA (for the W $\Delta \mathrm{C}$ mutant) or $\mathrm{W} \Delta \mathrm{F} \mathrm{Kv} 2.2$ or $\mathrm{W} \Delta \mathrm{C}$ $\mathrm{Kv} 2.2$ DNA (for the $\mathrm{W} \Delta \mathrm{F}-\mathrm{Y} \Delta \mathrm{T} \mathrm{Kv} 2.2$ and $\mathrm{W} \Delta \mathrm{C}-\mathrm{Y} \Delta \mathrm{T} \mathrm{Kv} 2.2$ clones, respectively). After gel purification of the desired round one fragments, the second round of PCR used Universal Forward and Universal Reverse as primers and the PCR products from the two round one reactions as both template and primers. In the initial cycle of the second round of PCR, the region of overlap between the two first round fragments was extended. During subsequent cycles these extended fragments were amplified by the Universal Forward and Reverse Primers. The final PCR product was cut with SalI and NcoI, gel-purified, and ligated into the pA2.2 construct that previously had been digested with SalI and NcoI. Clones identified as ones containing the desired mutations by restriction enzyme analysis were sequenced to confirm that (1) the correct substitutions had been made in the pore region, (2) no other mutations were introduced into the cassette during PCR amplification, and (3) introduction of the SalI-NcoI pore cassette occurred without frame shifts. In addition, wild-type Kv2.2 and each mutant clone was cut with 7-11 different restriction enzymes. The restriction digest patterns were com-

pared in each case to ensure that unexpected alterations had not taken place elsewhere in each mutant. RNA synthesis from the mutant clones was as described above for wild-type Kv2.1 and Kv2.2 clones.

Oocyte injection and recording. Fifty nanoliters of solution containing varying ratios of wild-type/mutant potassium channel RNA (0.002-0.3 $\mathrm{mg} / \mathrm{ml}$ ) were injected into stage VI defolliculated Xenopus oocytes prepared as described previously (Burger and Ribera, 1996). Oocytes were incubated at $18^{\circ} \mathrm{C}$, and voltage-activated potassium currents were recorded with standard two-electrode voltage clamp procedures (Axoclamp 2A amplifier, Axon Instruments, Foster City, CA) 1-2 d after injection. Voltage protocols and data analysis were accomplished with the pCLAMP suite of programs (Axon Instruments). Currents were sampled at $200 \mu \mathrm{sec}$ and leak-subtracted by a $\mathrm{P} / 4$ protocol. The electrode solution consisted of $3 \mathrm{M} \mathrm{KCl}$ and $10 \mathrm{~mm}$ HEPES, $\mathrm{pH}$ 7.4, whereas the bath contained Barth's solution [(in mM) $88 \mathrm{NaCl}, 1 \mathrm{KCl}, 0.33$ $\mathrm{Ca}\left(\mathrm{NO}_{3}\right)_{2}, 2.4 \mathrm{NaHCO}_{3}, 0.82 \mathrm{MgSO}_{4}$, and 5 Na-HEPES, pH 7.4]. Electrode resistance ranged between 0.1 and $0.5 \mathrm{M} \Omega$. Oocytes were not used if the holding current exceeded $-200 \mathrm{nA}$ at $-80 \mathrm{mV}$.

Data analysis. The binomial equation was used to calculate the fraction $\left(F_{\mathrm{i}}\right)$ of channels of a particular type $i$ :

$$
F_{i}=(4 ! /(4-i) ! i !) F_{\mathrm{WT}}^{(4-i)} F_{\mathrm{MUT}}^{i},
$$

where $F_{\mathrm{WT}}$ and $F_{\mathrm{MUT}}$ are the fractions of wild-type and mutant subunits. Discussion reviews the assumptions made in using the binomial equation (MacKinnon, 1991).

$\chi^{2}$ analysis (Bevington, 1969) was used to evaluate statistically the number of $\mathrm{W} \Delta \mathrm{C}-\mathrm{Y} \Delta \mathrm{T} \mathrm{Kv} 2.2$ mutant subunits required for a block that gave the best fit to the data. A reduced form of the $\chi^{2}$ statistic was used such that smaller $\chi^{2}$ values indicate better fits and $\chi^{2}<1$ indicates a fit to the data within $1 \mathrm{SD} . \chi^{2}$ analysis was performed only for wild-type $\mathrm{Kv} 2.2 / \mathrm{W} \Delta \mathrm{C}-\mathrm{Y} \Delta \mathrm{T}$ mixtures (i.e., for Table 3 and the limited data of Table 4 referring to the Kv2.2 mixtures), because the binomial equation cannot be used to predict the number of $\mathrm{W} \Delta \mathrm{C}-\mathrm{Y} \Delta \mathrm{T} \mathrm{Kv} 2.2$ subunits required to block Kv2.1 channels (wild-type Xenopus Kv2.1 and Kv2.2 may have different single-channel conductances). For the case of two $\mathrm{W} \Delta \mathrm{C}-\mathrm{Y} \Delta \mathrm{T}$ Kv2.2 mutant subunits required for a block, $\chi^{2}$ values were calculated without taking the order of mutant subunits in the tetramer into account (i.e., a channel with two mutant subunits side by side was considered equivalent to one with alternating wild-type and mutant subunits). Consideration of the order of mutant subunits in the heterotetramer gave a worse fit to the data, as demonstrated by the following. For two adjacent $\mathrm{W} \Delta \mathrm{C}-\mathrm{Y} \Delta \mathrm{T}$ mutant Kv2.2 subunits required for a block, the $\chi^{2}$ values are 1.34 and 5.53 for Tables 3 and 4 , respectively, whereas for alternating mutant subunits required for a block, the $\chi^{2}$ values are 4.0 and 10.81 for Tables 3 and 4, respectively. These compare with $\chi^{2}$ values of 0.26 and 


\begin{tabular}{llcc}
\hline \multicolumn{2}{l}{ Table 1. A double mutation in the pore region is required to create a dominant-negative Kv2.2 subunit } \\
Clone & Pore region sequence & $\begin{array}{l}\text { Functional as } \\
\text { homomultimer? }\end{array}$ & $\begin{array}{c}\text { Dominant- } \\
\text { negative? }\end{array}$ \\
\hline Kv2.2 & P A S F W W A T I T M T T V G Y G D I Y P & Yes & No \\
W $\Delta \mathrm{F}$ & P A F F F W A T T M T T V G Y G D I Y P & Yes & No \\
$\mathrm{W} \Delta \mathrm{C}$ & P A S F C W A T T M T T V G Y G D I Y P & No & No \\
$\mathrm{W} \Delta \mathrm{F}-\mathrm{Y} \Delta \mathrm{T}$ & P A S F F W A T I T M T T V G Y G D I T P & No & No \\
W $\Delta \mathrm{C}-\mathrm{Y} \Delta \mathrm{T}$ & P A S F C W A T I T M T T V G Y G D I T P & No & Yes \\
\hline
\end{tabular}

Although three of the mutants are nonfunctional when expressed as homomultimers, only the W $\Delta \mathrm{C}-\mathrm{Y} \Delta \mathrm{T}$ mutant shows dominant-negative properties. In the wild-type sequences, the substituted amino acids are in bold; the wild-type Xenopus Kv2.2 sequence is taken from Burger and Ribera (1996).

2.07 for Tables 3 and 4 in the case of two mutant subunits required for a block regardless of order.

\section{RESULTS}

\section{Creation of a dominant-negative mutant Kv2.2 subunit}

The major goal of this study was to determine whether heteromultimerization of different isotypes occurs within the Kv2 family. Accordingly, we constructed a dominant-negative Kv2 subunit with the following essential properties: (1) homomultimeric channels assembled from mutant subunits are not functional (negative), and (2) inclusion of less than three mutant subunits in a heterotetramer yields a nonf unctional channel (dominant). Previous work has shown that targeting the pore region is an effective strategy for generation of a Kv1 dominant-negative subunit (Taglialatela et al., 1994; Ribera, 1996; Ribera et al., 1996; Tinker et al., 1996). Mutations in the Xenopus Kv2.2 channel therefore were confined to the pore region. An advantage of this approach is that the pore has not been implicated as a region required for the coassembly of subunits.

The initial mutation made was a tryptophan-to-phenylalanine $(\mathrm{W} \Delta \mathrm{F})$ substitution in the putative pore region of Kv2.2 (Table 1). The analogous alteration in fly Kv1 results in a nonconducting potassium channel that still undergoes normal gating transitions (Perozo et al., 1993). In Xenopus Kv1.1 the tryptophan-tophenylalanine mutation gives rise to an efficient dominantnegative subunit (Ribera, 1996). Introduction of the $\mathrm{W} \Delta \mathrm{F}$ mutation into Xenopus Kv2.2 ( $\mathrm{W} \Delta \mathrm{F} \mathrm{Kv} 2.2)$, however, results in a subunit that, when injected into the oocyte, still induces ionic current although the current amplitude is reduced as compared with wild-type Kv2.2 expressed alone (Fig. 2C). Further, coinjection of wild-type Kv2.2 and $\mathrm{W} \Delta \mathrm{F} \mathrm{Kv} 2.2$ subunits into the oocyte does not lead to a reduction in current as compared with wildtype Kv2.2 expressed alone (Fig. 3B), indicating that the $\mathrm{W} \Delta \mathrm{F}$ Kv2.2 mutant subunit is neither negative or dominant.

On the basis of the suggestion of Dr. Rolf Joho (Baylor College of Medicine, Houston, Texas), we next mutated this tryptophan $\left(\operatorname{Trp}^{369}\right)$ to a cysteine (Table 1$)$, because this alteration in rat Kv2.1 leads to the creation of a subunit that does not form functional channels (Kurz et al., 1995). When expressed in the oocyte, homomultimeric $\mathrm{W} \Delta \mathrm{C} \mathrm{Kv} 2.2$ mutant channels are also nonf unctional (Table 1; Fig. 2D).

An initial assessment of the ability of the $\mathrm{W} \Delta \mathrm{C} \mathrm{Kv} 2.2$ mutant to act as a dominant-negative subunit is provided by coinjection with wild-type Kv2.2 RNA in a 1:1 ratio. We define the potency of a dominant-negative $\alpha$-subunit as the number of $\alpha$-subunits in a channel (i.e., 4) (MacKinnon, 1991; Liman et al., 1992) divided by the number of mutant subunits required to render a heteromultimer nonf unctional. If one, two, three, or four mutant subunits

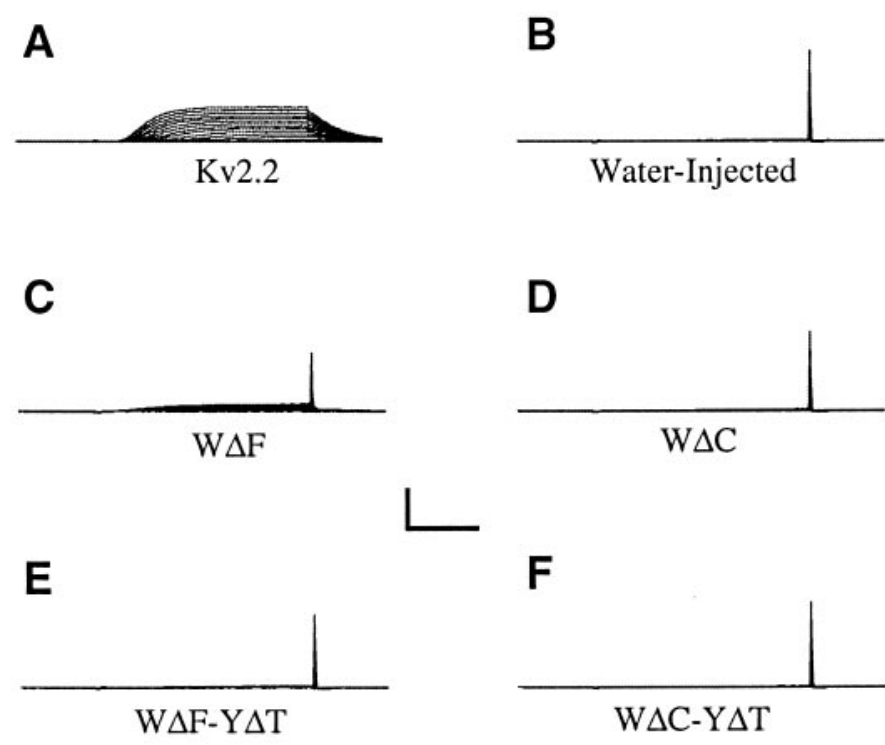

Figure 2. Amino acid substitutions in the pore region create nonfunctional Kv2.2 subunits. Currents are induced in the oocyte by the injection of $6.25 \mathrm{ng}$ of wild-type Kv2.2 RNA $(A)$ or $6.25 \mathrm{ng}$ of each of the Kv2.2 pore mutants $(C-F)$. Homomultimeric expression of $\mathrm{W} \Delta \mathrm{C} \mathrm{Kv} 2.2$, W $\Delta \mathrm{F}$ $\mathrm{Y} \Delta \mathrm{T} \mathrm{Kv} 2.2$, and $\mathrm{W} \Delta \mathrm{C}-\mathrm{Y} \Delta \mathrm{T} \mathrm{Kv} 2.2$ transcripts does not result in the formation of functional channels $(D-F)$, whereas injection of $\mathrm{W} \Delta \mathrm{F}$ Kv2.2 RNA $(C)$ induces currents with reduced amplitude as compared with wild-type Kv2.2. The injection of RNase-free water into the oocyte does not induce currents $(B)$. Currents were generated in response to $60 \mathrm{msec}$ voltage steps to potentials ranging from -50 to $+100 \mathrm{mV}$ from a holding potential of $-80 \mathrm{mV}$; leak-subtracted currents are shown (see Materials and Methods). Calibration: $3.5 \mu \mathrm{A}, 20 \mathrm{msec}$.

are required, the potencies are 4, 2, 1.33, and 1, respectively. We desire a potency of 2 or greater.

In our experiments the absolute amount of wild-type Kv2.2 RNA in each injection solution is kept constant by dilution either with RNase-free water or the appropriate mutant Kv2.2 RNA. A 1:1 mixture of wild-type and mutant transcripts injected into the oocyte results in the induction of smaller currents (Fig. 3C). Assuming that potassium channels are tetrameric, coexpression of wild-type and mutant subunits should lead to the formation of five channel classes: wild-type and mutant homomultimers and heteromultimeric channels containing one, two, or three mutant subunits. The binomial equation is used to predict the percentage of channels expected in each class for various ratios of wild-type/ mutant RNA (MacKinnon, 1991) (see equation in Materials and Methods). In the case of a 1:1 mixture of wild-type and mutant subunits, if two mutant subunits suffice to render heteromultimeric channels nonfunctional, $31.25 \%$ of the channels formed 


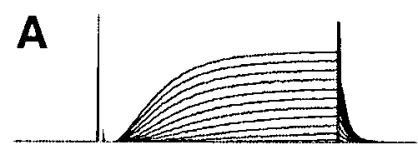

$\mathrm{Kv} 2.2$

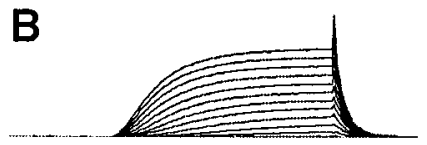

$\mathrm{Kv} 2.2+\mathrm{W} \Delta \mathrm{F}$

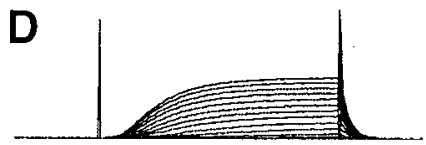

$\mathrm{Kv} 2.2+\mathrm{W} \Delta \mathrm{F}-\mathrm{Y} \Delta \mathrm{T}$

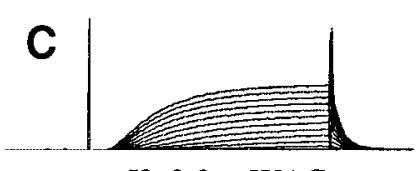

$\mathrm{Kv} 2.2+\mathrm{W} \Delta \mathrm{C}$

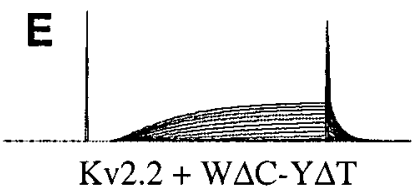

Figure 3. Double mutations in the Kv2.2 pore region are required for the construction of an efficient inhibitory subunit. Currents were recorded from oocytes injected with wild-type $\mathrm{Kv} 2.2$ transcripts alone $(A)$ or wild-type Kv2.2 RNA mixed with each pore mutant in a 1:1 ratio $(B-E)$. The amount of wild-type Kv2.2 RNA in each injection solution was kept constant. Coinjection of the $\mathrm{W} \Delta \mathrm{F}$ Kv2.2 subunit caused no reduction in current amplitude as compared with wild-type $\mathrm{Kv} 2.2$ currents $(B)$; coexpression of $\mathrm{W} \Delta \mathrm{C} \mathrm{Kv} 2.2$ and $\mathrm{W} \Delta \mathrm{F}-\mathrm{Y} \Delta \mathrm{T} \mathrm{Kv} 2.2$ with wild-type $\mathrm{Kv} 2.2$ led to an intermediate reduction in current amplitude $(C, D)$, and coinjection of $\mathrm{W} \Delta \mathrm{C}-\mathrm{Y} \Delta \mathrm{T} \mathrm{Kv} 2.2$ transcripts caused the greatest decrease in current size $(E)$. Currents were generated in response to $60 \mathrm{msec}$ voltage steps to potentials ranging from -50 to $+100 \mathrm{mV}$ from a holding potential of -80 $\mathrm{mV}$; leak-subtracted currents are shown (see Materials and Methods). Calibration: $3.5 \mu \mathrm{A}, 20 \mathrm{msec}$.

will conduct ionic current. Further, oocytes injected with the 1:1 mixture contain twice as much RNA and presumably twice the number of channels as those receiving wild-type transcripts alone. Accordingly, the current amplitude recorded from oocytes injected with the 1:1 mixture of wild-type and $\mathrm{W} \Delta \mathrm{C} \mathrm{Kv} 2.2$ subunits should be $63 \%$ of that recorded from oocytes injected only with wild-type Kv2.2 mRNA. The results of the mixing experiment (Table 2; Fig. 3) indicate, however, that more than two $\mathrm{W} \Delta \mathrm{C} \mathrm{Kv} 2.2$ subunits are required to suppress function in the heteromultimer. The $\mathrm{W} \Delta \mathrm{C} \mathrm{Kv} 2.2$ mutant thus has a potency of $<2$.

Further mutagenesis directed at the creation of a dominantnegative subunit was guided by comparison of the pore regions of the fly Kv1 and Xenopus Kv2.2 channels. In the Drosophila Kv1 gene in which the original tryptophan-to-phenylalanine mutation was made, the amino acid at position 449 is a threonine, whereas in Xenopus Kv2.2 the corresponding residue is a tyrosine $\left(\mathrm{Tyr}^{388}\right)$. So that the Xenopus mutant Kv2.2 clones would resemble more closely the fly Shaker $\mathrm{H} 4 \mathrm{~W}$-to-F mutant, $\mathrm{Tyr}^{388}$ was altered to threonine (following the suggestion of Dr. Rod MacKinnon, Rockefeller Institute, New York), generating the double mutants $\mathrm{W} \Delta \mathrm{F}-\mathrm{Y} \Delta \mathrm{T}$ Kv2.2 and $\mathrm{W} \Delta \mathrm{C}-\mathrm{Y} \Delta \mathrm{T}$ Kv2.2 (Table 1). When injected into the oocyte, neither $\mathrm{W} \Delta \mathrm{F}-\mathrm{Y} \Delta \mathrm{T}$ Kv2.2 nor W $\Delta \mathrm{C}-\mathrm{Y} \Delta \mathrm{T}$ Kv2.2 subunits induce the expression of ionic currents (see Fig. $2 E, F)$. They thus qualify as negative subunits. To provide an initial measure of the potency of each mutant clone, we coinjected wild-type Kv2.2 transcripts and each double mutant in a 1:1 ratio. Under these conditions, both $\mathrm{W} \Delta \mathrm{F}-\mathrm{Y} \Delta \mathrm{T}$ Kv2.2 and $\mathrm{W} \Delta \mathrm{C}-\mathrm{Y} \Delta \mathrm{T}$
Table 2. Only the W $\Delta \mathrm{C}-\mathrm{Y} \Delta \mathrm{T}$ mutant subunit acts in a dominantnegative manner

\begin{tabular}{|c|c|c|c|c|}
\hline \multirow[b]{2}{*}{ RNA injected } & \multicolumn{2}{|c|}{$\begin{array}{l}\text { Normalized amplitude of } \\
\text { recorded current }(\%)\end{array}$} & \multicolumn{2}{|c|}{$\begin{array}{l}\text { Expected am- } \\
\text { plitude if } i \text { sub- } \\
\text { units suffice for } \\
\text { block }\end{array}$} \\
\hline & & $(n)$ & $i=2$ & $i=3$ \\
\hline $\mathrm{Kv} 2.2$ & $100 \pm 3$ & $(45)$ & NA & NA \\
\hline $\mathrm{Kv} 2.2: \mathrm{W} \Delta \mathrm{F}$ & $121 \pm 15$ & (6) & 63 & 138 \\
\hline $\mathrm{Kv} 2.2: \mathrm{W} \Delta \mathrm{C}$ & $70 \pm 8$ & $(6)$ & 63 & 138 \\
\hline $\mathrm{Kv} 2.2: \mathrm{W} \Delta \mathrm{F}-\mathrm{Y} \Delta \mathrm{T}$ & $79 \pm 11$ & (11) & 63 & 138 \\
\hline $\mathrm{Kv} 2.2: \mathrm{W} \Delta \mathrm{C}-\mathrm{Y} \Delta \mathrm{T}$ & $60 \pm 6$ & (35) & 63 & 138 \\
\hline
\end{tabular}

Oocytes were injected with Xenopus Kv2.2 RNA alone or in a 1:1 ratio with each Kv2.2 mutant clone, as described in Materials and Methods. Coexpression of wild-type and mutant Xenopus Kv2.2 RNAs should lead to the formation of wildtype or mutant homomultimers and channels composed of one, two, or three mutant subunits. The binomial equation (MacKinnon, 1991) (see Materials and Methods) was used to calculate the percentage of each channel type expected to form and thus the percentage of wild-type current that would be measured if two or three mutant subunits suffice to render heteromultimeric channels nonf unctional. To normalize current amplitudes that varied between experimental days, we determined for each day the average current amplitude recorded from control oocytes injected with wild-type Kv2.2 RNA alone and then expressed the result recorded from each oocyte injected with a mixture of wild-type and mutant RNA as a fraction of the control current recorded that day. Control oocytes and those injected with a mixture of wild-type and mutant Kv2.2 RNAs were obtained from the same frog. Current amplitudes are taken at $+20 \mathrm{mV}$ and are given as means \pm SEM. NA, Not applicable.

Kv2.2 subunits cause a reduction in current amplitude as compared with wild-type Kv2.2 RNA injected alone (Fig. 3D,E). However, the $\mathrm{W} \Delta \mathrm{C}-\mathrm{Y} \Delta \mathrm{T} \mathrm{Kv} 2.2$ mutant subunit induces a greater reduction in functional expression (Table 2). This experiment suggests that the potency of the $\mathrm{W} \Delta \mathrm{F}-\mathrm{Y} \Delta \mathrm{T} \mathrm{Kv} 2.2$ mutant is less than that of the $\mathrm{W} \Delta \mathrm{C}-\mathrm{Y} \Delta \mathrm{T}$ mutant.

The potency of the $\mathrm{W} \Delta \mathrm{C}-\mathrm{Y} \Delta \mathrm{T} \mathrm{Kv} 2.2$ subunit is examined further by coinjecting different ratios of wild-type $\mathrm{Kv} 2.2 / \mathrm{W} \Delta \mathrm{C}$ $\mathrm{Y} \Delta \mathrm{T}$ mutant Kv2.2 transcripts into the oocyte. Adding increasing amounts of $\mathrm{W} \Delta \mathrm{C}-\mathrm{Y} \Delta \mathrm{T} \mathrm{Kv} 2.2 \mathrm{RNA}$ to the injection solution leads to a progressive decrease in the current amplitude as compared with that recorded from oocytes expressing wild-type Kv2.2 RNA alone (Fig. 4). Close to two mutant subunits render heteromultimeric channels nonfunctional (Table 3 ), indicating that the negative phenotype of the $\mathrm{W} \Delta \mathrm{C}-\mathrm{Y} \Delta \mathrm{T} \mathrm{Kv} 2.2$ subunit is dominant and has a potency of 2 , as desired.

The amplitude of the current expressed by oocytes injected with a mixture of wild-type Kv2.2 and $\mathrm{W} \Delta \mathrm{C}-\mathrm{Y} \Delta \mathrm{T}$ mutant transcripts as compared with that induced in oocytes containing wild-type Kv2.2 RNA alone was measured at a single voltage. Therefore, changes in the conductance-voltage relationships for currents recorded from oocytes injected with wild-type Kv2.2 RNA versus those injected with wild-type plus dominant-negative mutant transcripts could complicate interpretation of the data. To ensure that the decrease in current amplitude recorded from coinjected oocytes is not attributable to an alteration in voltagedependent properties of activation, we compared the conductance-voltage relationship for wild-type currents and those recorded from oocytes expressing a wild-type/mutant mixture. The voltage-dependent properties of activation are similar for oocytes injected with either wild-type or wild-type/W $\Delta \mathrm{C}-\mathrm{Y} \Delta \mathrm{T}$ transcripts (see Fig. 6C,D). This suggests that channels containing mutant Kv2.2 subunits do not demonstrate altered voltage-sensing properties. 
A

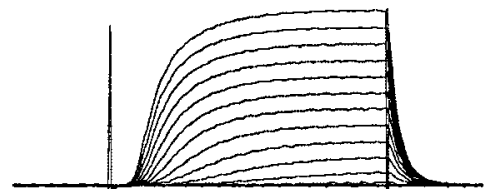

$\mathrm{Kv} 2.2$
B

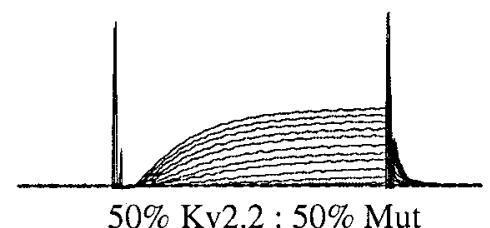

C

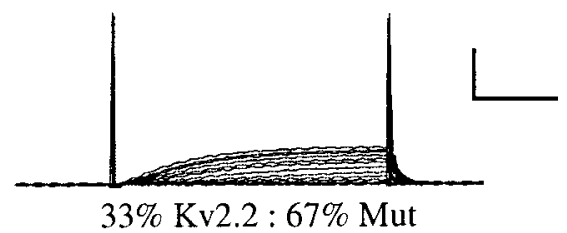

Figure 4. W $\Delta \mathrm{C}-\mathrm{Y} \Delta \mathrm{T} \mathrm{Kv} 2.2$ behaves as expected for a dominant-negative subunit. Oocytes were injected with wild-type Kv2.2 RNA $(A)$ or two different ratios of wild-type and $\mathrm{W} \Delta \mathrm{C}$-Y $\mathrm{Y}$ T mutant transcripts $(B, C)$. The amount of wild-type RNA in each injection solution was kept constant. Increasing the ratio of mutant to wild-type RNA led to the induction of smaller currents despite the increasing amount of total RNA injected into the oocyte. Currents were generated in response to $60 \mathrm{msec}$ voltage steps to potentials ranging from -50 to $+100 \mathrm{mV}$ from a holding potential of $-80 \mathrm{mV}$; leak-subtracted currents are shown (see Materials and Methods). Calibration: $2.7 \mu \mathrm{A}, 20 \mathrm{msec}$.

Table 3. Potency of the $\mathrm{W} \Delta \mathrm{C}-\mathrm{Y} \Delta \mathrm{T}$ dominant-negative subunit

\begin{tabular}{|c|c|c|c|c|c|}
\hline \multirow{2}{*}{$\begin{array}{l}\text { Ratio of } \\
\mathrm{Kv} 2.2: \mathrm{W} \Delta \mathrm{C}-\mathrm{Y} \Delta \mathrm{T} \text { injected }\end{array}$} & \multicolumn{2}{|c|}{$\begin{array}{l}\text { Normalized amplitude of } \\
\text { recorded current }(\%)\end{array}$} & \multicolumn{3}{|c|}{$\begin{array}{l}\text { Expected amplitude if } i \text { subunits } \\
\text { suffice for block }\end{array}$} \\
\hline & & $(n)$ & $i=1$ & $i=2$ & $i=3$ \\
\hline & & & $\chi^{2}=2.1$ & $\chi^{2}=0.26$ & $\chi^{2}=8.41$ \\
\hline 1:0 & $100 \pm 3$ & $(45)$ & NA & NA & NA \\
\hline $0: 1$ & $2 \pm 0.4$ & (25) & NA & NA & NA \\
\hline $1: 0.5$ & $65 \pm 10$ & (12) & 30 & 89 & 34 \\
\hline $1: 1$ & $60 \pm 6$ & (35) & 12 & 63 & 138 \\
\hline $1: 2$ & $37 \pm 8$ & (16) & 4 & 33 & 122 \\
\hline
\end{tabular}

As few as two mutant subunits suffice to render resultant Kv2.2 channels nonfunctional. Currents were recorded from oocytes that had been injected with a constant amount of wild-type Xenopus Kv2.2 RNA and either no or varying amounts of $\mathrm{W} \Delta \mathrm{C}-\mathrm{Y} \Delta \mathrm{T}$ mutant Kv2.2 RNA; W $\Delta \mathrm{C}-\mathrm{Y} \Delta \mathrm{T}$ RNA also was injected by itself. For a given ratio of Kv2.2:W $\Delta \mathrm{C}-\mathrm{Y} \Delta \mathrm{T}$ RNA injected, the binomial equation was used to determine the percentage of homo- or heteromultimeric channels in each class and thus to predict the percentage of wild-type Kv2.2 current that would be measured if one, two, or three mutant subunits suffice to prevent formation of functional channels. Current amplitudes, which were normalized as described in Table 2, are taken at $+20 \mathrm{mV}$ and are given as means \pm SEM. $\chi^{2}$ analysis (see Materials and Methods) was used to determine the number of $\mathrm{W} \Delta \mathrm{C}-\mathrm{Y} \Delta \mathrm{T}$ Kv2.2 mutant subunits required for block that gave the best fit to the data. This analysis indicates that two mutant subunits give the best fit to the data. NA, Not applicable.

\section{Coexpression of wild-type Kv2.1 and dominant- negative Kv2.2 subunits}

If Kv2.1 and Kv2.2 subunits coassemble, inclusion of the $\mathrm{W} \Delta \mathrm{C}$ $\mathrm{Y} \Delta \mathrm{T}$ mutant Kv2.2 subunit in Kv2.1-containing channels should result in a reduction in current as compared with that induced by homomultimeric Kv2.1 channels. Thus, wild-type Kv2.1 and dominant-negative Kv2.2 subunits were coexpressed in the oocyte.

Currents induced in the oocyte by injection of wild-type Kv2.1 transcripts are much larger, however, than those induced by an equal amount of wild-type Kv2.2 RNA (data not shown). An appropriate normalization thus was required for examination of the formation of Kv2.1 homomultimers, Kv2.2 homomultimers, and possible Kv2.1/Kv2.2 heteromultimeric channels. For these experiments, normalization was achieved on the basis of functional expression rather than on the amount of RNA injected. The amount of wild-type Kv2.1 injected into the oocyte was an order of magnitude less than that of wild-type Kv2.2 or the $\mathrm{W} \Delta \mathrm{C}-\mathrm{Y} \Delta \mathrm{T} \mathrm{Kv} 2.2$ mutant so as to obtain equivalent functional expression of the two channel isotypes (Fig. 5A1,B1). The expectation of equivalent functional expression was verified by normalizing the current amplitudes obtained to both wild-type Kv2.1 alone and wild-type Kv2.2 alone (Table 4). Coexpression of wild-type Kv2.1 and dominant-negative mutant Kv2.2 transcripts in either a $1: 1$ or a 1:4 ratio results in a reduction in current amplitude as compared with wild-type Kv2.1 or wild-type Kv2.2 RNA injected alone (Table 4; Fig. 5C2,C3), implying the forma- tion of heteromultimeric channels in which the Kv2.2 mutant suppresses wild-type Kv2.1 subunit function.

Although the results are consistent with the dominant-negative Kv2.2 subunit poisoning Kv2.1 as well as Kv2.2-containing channels, other explanations exist. For example, the decrease in current size recorded from coinjected (wild-type/mutant) oocytes could be attributable to saturation of the translation machinery of the oocyte with higher RNA doses. However, larger currents are induced in cells expressing a mixture of wild-type Kv2.1 and Kv2.2 transcripts as compared with those recorded from oocytes expressing only a single RNA species (Table 4; Fig. 5A2,A3). Alternatively, the conductance-voltage relationships for wildtype Kv2.1 currents and those recorded from oocytes expressing a 1:1 ratio of wild-type Kv2.1/W $\Delta \mathrm{C}-\mathrm{Y} \Delta \mathrm{T} \mathrm{Kv} 2.2$ subunits could be altered. However, these are similar (Fig. $6 A, B$ ). Thus, the reduction in current size seen in coinjected oocytes is not attributable to any difference in the voltage-dependent properties of activation for wild-type Kv2.1 or wild-type Kv2.1/W $\Delta \mathrm{C}-\mathrm{Y} \Delta \mathrm{T} \mathrm{Kv} 2.2$ currents. Further, normalization was according to current amplitude and not the number of channels; the single-channel conductances of Xenopus Kv2.1 and Kv2.2 heteromultimers are not known and might be different. However, similar results were obtained whether the normalization was done for Kv2.1 or Kv2.2 current amplitudes (Table 4).

The dominant-negative Kv2.2 subunit differs from wild-type $\mathrm{Kv} 2.2$ at residues located in the pore region rather than regions implicated in heteromultimerization. Thus, we conclude that 
A1

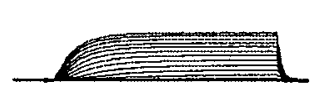

$\mathrm{Kv} 2.1$

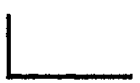

A2

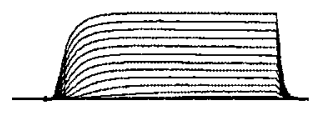

Kv2.1: Kv2.2 1:1

A3

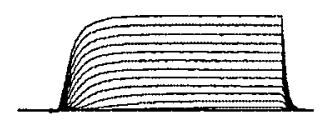

Kv2.1 : Kv2.2 1:4
B1

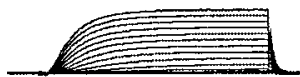

$\mathrm{Kv} 2.2$

B2

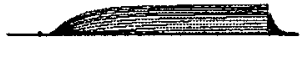

$\mathrm{Kv} 2.2$ : W $\Delta \mathrm{C}-\mathrm{Y} \Delta \mathrm{T} 1: 1$

B3

$\mathrm{K} v 2.2: \mathrm{W} \Delta \mathrm{C}-\mathrm{Y} \Delta \mathrm{T} 1: 4$
C1

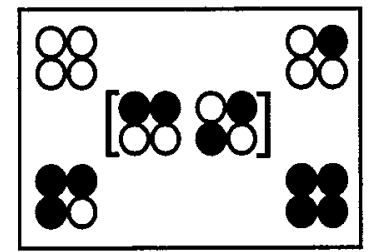

C2

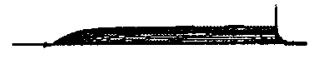

Kv2.1: W $\Delta \mathrm{C}-\mathrm{Y} \Delta \mathrm{T} 1: 1$

Figure 5. Kv2.1 and Kv2.2 subunits coassemble. Oocytes were injected with wild-type Kv2.1 alone (A1), wild-type Kv2.2 alone (B1), a functionally equal amount of wild-type Kv2.1 and Kv2.2 RNA ( $A 2)$, four times the functional amount of wild-type Kv2.2 as Kv2.1 RNA ( $A 3$ ), an equal amount of wild-type Kv2.2 and dominant-negative Kv2.2 RNA (B2), four times the amount of dominant-negative Kv2.2 RNA as wild-type Kv2.2 transcripts (B3), a functionally equal amount of wild-type Kv2.1 RNA and dominant-negative Kv2.2 RNA (C2), or four times the functionally equivalent amount of dominant-negative Kv2.2 transcripts as wild-type Kv2.2 RNA (C3). The dominant-negative Kv2.2 subunit causes a similar reduction in current amplitude when it is coexpressed with either wild-type Kv2.1 or wild-type Kv2.2 subunits (compare $B 2$ and $C 2, B 3$ and $C 3$ ) despite the increased amount of total RNA injected into the oocyte. Coexpression of wild-type Kv2.1 and Kv2.2 subunits leads to the induction of larger currents $(A 2, A 3)$. In the diagram (C1), open circles represent wild-type Kv2.1 subunits, and filled circles represent dominant-negative Kv2.2 subunits. The heteromultimeric Kv2.1/ dominant-negative Kv2.2 channels represented in brackets indicate two possible arrangements for a heterotetramer containing two mutant subunits. Currents were generated in response to $160 \mathrm{msec}$ voltage steps to potentials ranging from -50 to $+100 \mathrm{mV}$ from a holding potential of $-80 \mathrm{mV}$; leak-subtracted currents are shown (see Materials and Methods). Calibration: $3.6 \mu \mathrm{A}, 80 \mathrm{msec}$.

Kv2.1 and Kv2.2 subunits are able to form heteromultimeric channels.

\section{DISCUSSION}

The dominant-negative $\mathrm{W} \Delta \mathrm{C}-\mathrm{Y} \Delta \mathrm{T} \mathrm{Kv} 2.2$ subunit, which differs from wild-type Kv2.2 at only two pore residues, was used to probe Kv2.1/Kv2.2 subunit heteromultimerization. We established the potency of the $\mathrm{W} \Delta \mathrm{C}-\mathrm{Y} \Delta \mathrm{T}$ subunit by coexpressing various ratios of wild-type and dominant-negative mutant Kv2.2 transcripts in oocytes and measuring the amplitudes of residual currents as compared with those induced by wild-type Kv2.2 transcripts expressed alone. The results indicate that Xenopus Kv2.1 and Kv2.2 subunits can form heteromultimeric channels in a heterologous expression system.

The binomial equation was used to calculate the percentage of channels in each class expected for a particular ratio of wild-type/ dominant-negative mutant RNA and thus the current amplitude predicted for a mutant subunit of a given potency. Use of the binomial distribution in this manner involves the following five assumptions: (1) each channel consists of four $\alpha$-subunits and no auxiliary subunits, (2) for each RNA species the number of subunits synthesized and channels formed is linearly dependent on the amount of RNA injected, (3) there is no preferential coassembly of wild-type and mutant subunits into either homo- or heteromultimeric channels, (4) when coassembled into hetero- multimeric channels, mutant subunits either have no effect on conductance or suppress function completely, and (5) the order of subunits in a heterotetrameric channel is not relevant (see Fig. $5 C 1$ and Materials and Methods). The first assumption has been tested in other studies (MacKinnon, 1991; Liman et al., 1992). To maximize the validity of the second assumption, we kept the final concentrations of all RNAs $(0.002-0.3 \mathrm{mg} / \mathrm{ml})$ in a range that should not saturate the translation machinery of the oocyte. However, even with these efforts the system showed nonlinearity (e.g., Table 4). The third assumption is reasonable, given that all mutations were targeted to the pore region and that assembly domains have been located elsewhere (Li et al., 1992; Shen et al., 1993; Babila et al., 1994; Hopkins et al., 1994; Lee et al., 1994; Shen and Pfaffinger, 1995). The fourth assumption has been examined previously at the single-channel level and found to be valid for dominant-negative Kv1.1 subunits (Ribera et al., 1996).

Although the assumptions that have been made are reasonable, alternate tests of the potency of the dominant-negative Kv2.2 subunit would be useful and were sought. We attempted to engineer either a pharmacological tag (sensitivity to Agitoxin $_{2}$; Gross and MacKinnon, 1994) or a functional tag (fast inactivation; Lu and Miller, 1995) into the mutant subunit. However, neither approach yielded a subunit that was either functional or distinguishable on the basis of the desired engineered tag. 


\begin{tabular}{|c|c|c|c|}
\hline \multirow[b]{2}{*}{ RNA condition } & & \multicolumn{2}{|c|}{ Percentage of current amplitude } \\
\hline & & $\begin{array}{l}\text { Normalized to } \\
\text { Kv2.1 alone }\end{array}$ & $\begin{array}{l}\text { Normalized to } \\
\text { Kv } 2.2 \text { alone }\end{array}$ \\
\hline I. Single RNA & $(n)$ & & \\
\hline a) Kv2.1 & (39) & $100 \pm 5$ & $101 \pm 6$ \\
\hline b) Kv2.2 & (31) & $100 \pm 5$ & $100 \pm 6$ \\
\hline c) $\mathrm{W} \Delta \mathrm{C}-\mathrm{Y} \Delta \mathrm{T}$ & (20) & $0 \pm 0$ & $0 \pm 0$ \\
\hline II. 1:1 RNA mixtures & $(n)$ & & \\
\hline a) Kv2.1:Kv2.2 & (33) & $149 \pm 13$ & $147 \pm 13$ \\
\hline b) Kv2.1:W $\Delta \mathrm{C}-\mathrm{Y} \Delta \mathrm{T}$ & (34) & $52 \pm 5$ & $47 \pm 6$ \\
\hline c) $\mathrm{Kv} 2.2: \mathrm{W} \Delta \mathrm{C}-\mathrm{Y} \Delta \mathrm{T}$ & (29) & $53 \pm 5$ & $53 \pm 4$ \\
\hline III. 1:4 RNA mixtures & $(n)$ & & \\
\hline a) Kv2.1:Kv2.2 & (31) & $153 \pm 11$ & $151 \pm 11$ \\
\hline b) Kv2.1:W $\Delta \mathrm{C}-\mathrm{Y} \Delta \mathrm{T}$ & (32) & $28 \pm 6$ & $27 \pm 6$ \\
\hline c) $\mathrm{Kv} 2.2: \mathrm{W} \Delta \mathrm{C}-\mathrm{Y} \Delta \mathrm{T}$ & (22) & $35 \pm 3$ & $38 \pm 4$ \\
\hline
\end{tabular}

Coexpression of dominant-negative Kv2.2 subunits with either wild-type Kv2.1 or Kv2.2 subunits leads to a similar reduction in current. In contrast, coinjection of wild-type Kv2.1 and Kv2.2 transcripts into the oocyte results in the induction of larger currents compared with either channel isoform injected alone. Current amplitudes are taken at $+20 \mathrm{mV}$ and are given as means \pm SEM. To obtain equivalent functional expression of Xenopus Kv2.1 and Kv2.2 channels, the amount of Xenopus Kv2.2 RNA injected was an order of magnitude larger than that of Kv2.1 RNA. To account for this difference, we normalized current amplitudes recorded from oocytes injected with Kv2.1 RNA-containing solutions to the average current amplitude recorded from all oocytes injected with Kv2.2 RNA alone, and we normalized currents recorded from oocytes injected with Kv2.2 RNA-containing solutions to the average Kv2.1 current amplitude. For a limited set of data (all of the wild-type $\mathrm{Kv} 2.2: \mathrm{W} \Delta \mathrm{C}-\mathrm{Y} \Delta \mathrm{T}$ mixtures), $\chi^{2}$ analysis (see Materials and Methods) was used to determine the number of W $\Delta \mathrm{C}-\mathrm{Y} \Delta \mathrm{T} \mathrm{Kv} 2.2$ mutant subunits required for block that gave the best fit to the data. $\chi^{2}$ values are as follows: $\chi^{2}=7.85$ for one mutant subunit required for block, $\chi^{2}=2.07$ for two mutant subunits required for block and $\chi^{2}=23.77$ for three mutant subunits required for block. The $\chi^{2}$ value for two mutant subunits required for block gives the best fit to the data.

Several different mechanisms can account for the dominantnegative phenotype of the $\mathrm{W} \Delta \mathrm{C}-\mathrm{Y} \Delta \mathrm{T}$ Kv2.2 mutant. For example, channels containing a W $\Delta \mathrm{C}-\mathrm{Y} \Delta \mathrm{T} \mathrm{Kv} 2.2$ subunit could be locked permanently in an inactivated state, as is the case for the fly Kv1.1 tryptophan-to-phenylalanine mutant (Young et al., 1997). Alternatively, conduction through the pore of $\mathrm{W} \Delta \mathrm{C}-\mathrm{Y} \Delta \mathrm{T}$ Kv2.2-containing channels could be blocked, or the dominantnegative Kv2.2 mutant could prevent wild-type Kv2.2 subunits from reaching the surface. Our data, however, do not allow us to distinguish between these possibilities.

Although the structural basis of Kv2 subunit coassembly has not yet been defined, studies that use Kv1 isotypes have implicated N-terminal regions (Li et al., 1992; Shen et al., 1993; Babila et al., 1994; Hopkins et al., 1994; Lee et al., 1994; Shen and Pfaffinger, 1995). Similarly, Xu et al. (1995) have demonstrated an association between the N-terminal portions of the fly Shab and rat Kv2.1 genes. VanDongen et al. (1990), however, reported that a truncated rat Kv2.1 subunit, missing 139 amino acids from the $\mathrm{N}$ terminus and 318 amino acids from the $\mathrm{C}$ terminus, forms homomultimers for which the functional properties resemble those of the wild-type rat Kv2.1 channel. Thus, for Kv2 channels, regions other than the $\mathrm{N}$ and $\mathrm{C}$ termini may contribute to subunit coassembly. In addition, Kv2.1 subunits appear to coassemble with members of the Kv6, Kv8, and Kv9 subfamilies (Hugnot et al., 1996; Post et al., 1996; Patel et al., 1997; Salinas et al., 1997a,b) as well as the Kv2.3r subunit (Castellano et al., 1997).

Our work showing that Xenopus Kv2.1 and Kv2.2 subunits form heteromultimeric channels in the oocyte is intriguing, given that rat Kv2.1 and Kv2.2 channels localize to distinct areas within a neuron (Trimmer, 1991; Hwang et al., 1993; Maletic-Savatic et al., 1995; Rhodes et al., 1995). The apparent discrepancy between the results obtained in the two systems may be attributable to differences between the Xenopus and rat Kv2 clones. However, Scan- nevin et al. (1996) have characterized a domain within the rat Kv2.1 polypeptide that is necessary for the correct localization of the Kv2.1 channel in polarized Madin-Darby canine kidney (MDCK) cells, a cell line used to model membrane protein targeting. In MDCK cells, rat Kv2.1 localizes to the lateral cell membrane, which correlates with its somatodendritic disposition in neurons. Although the region identified by Scannevin et al. (1996) is overall only $46 \%$ identical between rat and Xenopus Kv2.1 (157 amino acid identity over 341 residues), there are 14 stretches within this region in which the percentage of identity is $75 \%$ or greater over at least four consecutive residues. Further, preliminary data suggest that localization of Xenopus Kv2.1 in MDCK cells is similar to that of the rat Kv2.1 channel ( $R$. Scannevin, personal communication). These results suggest that Xenopus Kv2.1 polypeptides also would be localized selectively in situ.

Taken together with our demonstration of Kv2.1/Kv2.2 coassembly in the oocyte, the available evidence suggests that other mechanisms account for the lack of detection of heteromultimeric $\mathrm{Kv} 2$ channels in vivo. For example, Kv2.1/Kv2.2 heteromultimers might be present at very low, and thus undetectable, levels in the neuronal membrane. Another reason for the lack of in situ heteromultimer detection might be restricted spatial and temporal expression of the Kv2.1 and Kv2.2 channel subunits. MaleticSavatic et al. (1995) have found that, in the developing rat hippocampus, Kv2.1 polypeptides are expressed first, followed $4 \mathrm{~d}$ later by Kv2.2. Kv2.1 and Kv2.2 channels also might interact with different cytoskeletal elements, allowing for discrete potassium channel localization. In rat brain, for instance, Kv2.1 polypeptides are found in tight clusters in the neuronal membrane, whereas Kv2.2 proteins appear in a more diffuse pattern (Trimmer et al., 1991; Hwang et al., 1993; Maletic-Savatic et al., 1995; Rhodes et al., 1995). The localization domain identified by Scannevin et al. 
A

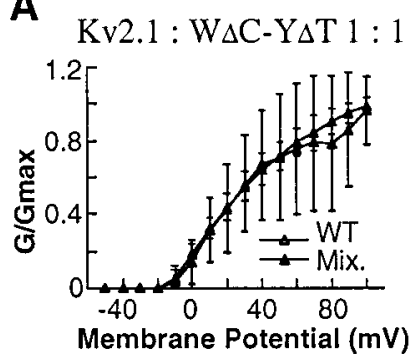

B
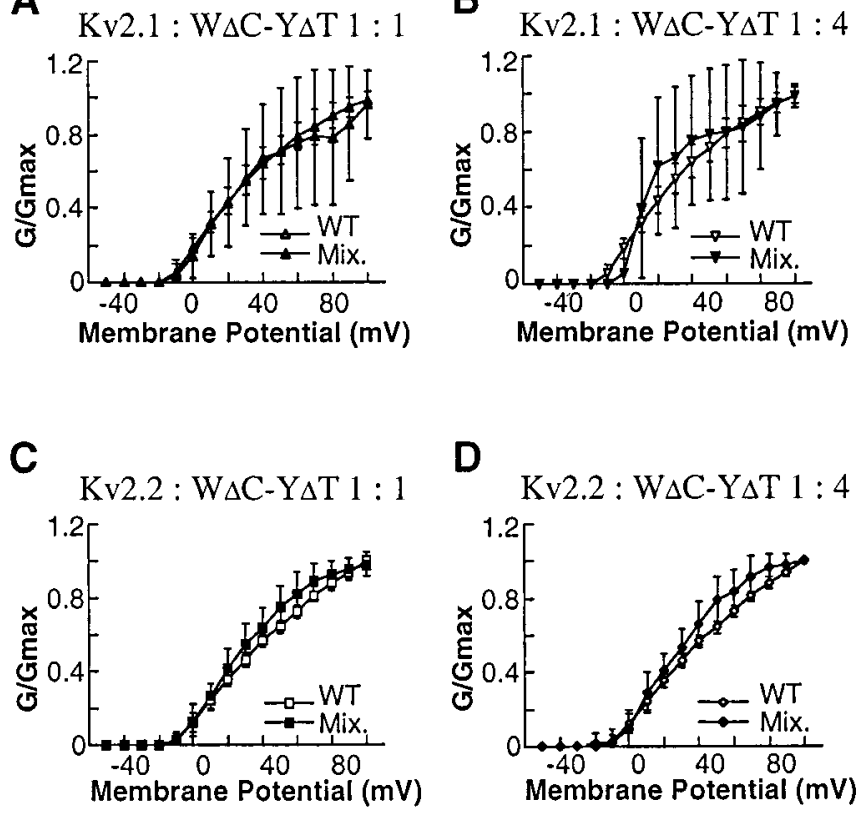

Figure 6. The conductance-voltage relations for wild-type Kv2.1 or wild-type Kv2.2 subunits expressed alone or in combination with $\mathrm{W} \Delta \mathrm{C}$ $\mathrm{Y} \Delta \mathrm{T}$ mutant or wild-type Kv2.1 and Kv2.2 subunits are similar. Conductance-voltage relations were plotted for wild-type Kv2.1 subunits alone or in combination with different ratios of dominant-negative mutant Kv2.2 subunits $(A, B)$. Similar graphs were derived for wild-type Kv2.2 subunits injected into the oocyte alone or with different ratios of $\mathrm{W} \Delta \mathrm{C}-\mathrm{Y} \Delta \mathrm{T} \mathrm{Kv} 2.2$ mutant subunits $(C, D)$. The steady-state activation properties of the wild-type Kv2.1 or Kv2.2 current and the current remaining with the coexpression of either wild-type Kv2.1 or wild-type Kv2.2 and different ratios of the mutant Kv2.2 subunit are similar. This is the result expected if the subunit acts in a dominant-negative manner or does not demonstrate altered voltage-sensing properties. Symbols are mean values; error bars indicate SD. The SD bars are large for wild-type/W $\Delta \mathrm{C}-\mathrm{Y} \Delta \mathrm{T}$ Kv2.2 mixtures (e.g., $A, B$ ). In these cases the current amplitudes are small, and the endogenous currents of the oocyte presumably contribute substantially to the overall current. The numbers of oocytes range from 17 to 39 .

(1996), which targets rat Kv2.1 to the lateral membrane in MDCK cells, also appears sufficient to induce Kv2.1 clustering in this cell line, suggesting its requirement for this distinctive localization pattern. Potential differential modifications of Kv2.1 and Kv2.2 subunits in situ also might prevent subunit coassembly between different members of the Kv2 subfamily. For example, Patton et al. (1997) have shown that transcripts encoded by the squid Kv2 channel are modified by RNA editing. Similarly, posttranscriptional and/or post-translational modification of subunits might influence the heteromultimerization of different Kv2 subunits.

What are the functional implications of the failure to detect Kv2.1/Kv2.2 heteromultimers in situ? First, each channel type could demonstrate distinctive, labile functional properties. In heterologous expression systems, both Kv2.1 and Kv2.2 homomeric channels generate sustained delayed rectifier-type currents that appear functionally similar (Hwang et al., 1992; Shi et al., 1994; Burger and Ribera, 1996; this study). Further, under the conditions in which Kv2.1/Kv2.2 heteromultimers form, only minor functional differences are noted between the homo- and heteromultimeric Kv2 channels (see, for example, Fig. 1). Accordingly, the expression of only homomultimeric functionally similar channel complexes in vivo may allow for the subunit-specific modulation of Kv2.1 versus Kv2.2 channels by either additional subunits or enzymes capable of interaction with one, but not the other, homotetramer. In the Xenopus Kv2 subfamily, for instance, Kv2.2 has a unique cAMP phosphorylation site at amino acid 14 and a unique tyrosine phosphorylation site at amino acid 512 . Second, inclusion of Kv2.2 subunits in Kv2.1-containing complexes might disrupt the characteristic clustering of Kv2.1 channels seen in situ (Trimmer et al., 1991; Hwang et al., 1993; Maletic-Savatic et al., 1995; Rhodes et al., 1995). These Kv2.1 channel clusters might be crucial for correct neuronal function.

Future work will elucidate both the domains required for the coassembly of subunits encoded by members of the Kv2 subfamily and the mechanisms involved in the segregated localization of Kv2 polypeptides. These studies will allow for a greater understanding of the mechanisms that allow voltage-gated potassium channels to play multiple roles in the acquisition and maintenance of electrical excitability in specific neuronal compartments.

\section{REFERENCES}

Babila T, Muscucci A, Wang H, Weaver FE, Koren G (1994) Assembly of mammalian voltage-gated potassium channels: evidence for an important role of the first transmembrane segment. Neuron 12:615-626.

Bevington PR (1969) Data reduction and error analysis for the physical sciences. New York: McGraw-Hill.

Burger C, Ribera AB (1996) Xenopus spinal neurons express Kv2 potassium channel transcripts during embryonic development. J Neurosci 16:1412-1421.

Castellano A, Chiara MD, Mellstrom B, Molina A, Monje F, Naranjo JR, Lopez-Barneo J (1997) Identification and functional characterization of a $\mathrm{K}^{+} \alpha$-channel subunit with regulatory properties specific to brain. J Neurosci 17:4652-4661.

Chandy KG (1991) Simplified gene nomenclature [letter]. Nature 352:26.

Chen ML, Hoshi T, Wu CF (1996) Heteromultimeric interactions among K ${ }^{+}$channel subunits from Shaker and eag families in Xenopus oocytes. Neuron 17:535-542.

Christie MJ, North RA, Osborne PB, Douglass J, Adelman JP (1990) Heteropolymeric potassium channels expressed in Xenopus oocytes from cloned subunits. Neuron 3:405-411.

Covarrubias M, Wei AA, Salkoff L (1991) Shaker, Shal, Shab, and Shaw express independent $\mathrm{K}^{+}$current systems. Neuron 7:763-773.

Gross A, MacKinnon R (1994) Transfer of the scorpion toxin receptor to an insensitive channel. Neuron 13:961-966.

Hollmann M, Maron C, Heinemann S (1994) N-glycosylation site tagging suggests a three transmembrane domain topology for the glutamate receptor GluR1. Neuron 13:1331-1343.

Hopkins WF, Demas V, Tempel BL (1994) Both N- and C-terminal regions contribute to the assembly and functional expression of homoand heteromultimeric voltage-gated $\mathrm{K}^{+}$channels. J Neurosci 14:1385-1393.

Hugnot JP, Salinas M, LeSage F, Guillemare E, De Weile J, Heurteaux C, Mattei MG, Lazdunski M (1996) Kv8.1, a new neuronal potassium channel with specific inhibitory properties towards Shab and Shaw channels. EMBO J 15:3322-3331.

Hwang PM, Glatt CE, Bredt DS, Yellen G, Snyder SH (1992) A novel $\mathrm{K}^{+}$channel with unique localization in mammalian brain: molecular cloning and characterization. Neuron 8:473-481.

Hwang PM, Fotuhi M, Bredt DS, Cunningham AM, Snyder SH (1993) Contrasting immunohistochemical localizations in rat brain of two novel $\mathrm{K}^{+}$channels of the Shab subfamily. J Neurosci 13:1569-1576.

Isacoff EY, Jan YN, Jan LY (1990) Evidence for the formation of heteromultimeric potassium channels in Xenopus oocytes. Nature 345:530-534.

Kurz LL, Zuhlke RD, Zhang HJ, Joho RH (1995) Side-chain accessibilities in the pore of a $\mathrm{K}^{+}$channel probed by sulfhydryl-specific reagents after cysteine-scanning mutagenesis. Biophys J 68:900-905.

Lee TE, Philipson LH, Kuznetsov A, Nelson DJ (1994) Structural determinant for assembly of $\mathrm{K}^{+}$channels. Biophys J 66:667-673.

Li M, Jan YN, Jan LY (1992) Specification of subunit assembly by the hydrophilic amino-terminal domain of the Shaker potassium channel. Science 257:1225-1230.

Liman ER, Tytgat J, Hess P (1992) Subunit stoichiometry of a mammalian $\mathrm{K}^{+}$channel determined by construction of multimeric cDNAs. Neuron 9:861-871. 
Lu Q, Miller C (1995) Silver as a probe of pore-forming residues in a potassium channel. Science 268:304-307.

MacKinnon R (1991) Determination of the subunit stoichiometry of a voltage-activated potassium channel. Nature 350:232-235.

Maletic-Savatic M, Lenn NJ, Trimmer JS (1995) Differential spatiotemporal expression of $\mathrm{K}^{+}$channel polypeptides in rat hippocampal neurons developing in situ and in vitro. J Neurosci 15:3840-3851.

Patel AJ, Lazdunski M, Honore E (1997) Kv2.1/Kv9.3, a novel ATPdependent delayed rectifier $\mathrm{K}^{+}$channel in oxygen-sensitive pulmonary artery myocytes. EMBO J 16:6615-6625.

Patton DE, Silva T, Bezanilla F (1997) RNA editing generates a diverse array of transcripts encoding squid $\mathrm{Kv} 2 \mathrm{~K}^{+}$channels with altered functional properties. Neuron 19:711-722.

Perozo E, MacKinnon R, Bezanilla F, Stefani E (1993) Gating currents from a nonconducting mutant reveal open-closed conformations in Shaker $\mathrm{K}^{+}$channels. Neuron 11:353-358.

Post MA, Kirsch GE, Brown AM (1996) Kv2.1 and electrically silent Kv6.1 potassium channel subunits combine and express a novel current. FEBS Lett 399:177-182.

Rhodes KJ, Keilgaugh SA, Barrezueta NX, Lopez KL, Trimmer JS (1995) Association and co-localization of $\mathrm{K}^{+}$channel $\alpha$ - and $\beta$-subunit polypeptides in rat brain. J Neurosci 15:5360-5371.

Ribera AB (1996) Homogeneous development of electrical excitability via heterogeneous ion channel expression. J Neurosci 16:1123-1130.

Ribera AB, Pacioretty LM, Taylor RS (1996) Probing molecular identity of native single potassium channels by overexpression of dominant negative subunits. Neuropharmacology 35:1007-1016.

Ruppersberg JP, Schroter KH, Sakmann B, Stocker M, Sewing S, Pongs O (1990) Heteromultimeric channels formed by rat brain potassiumchannel proteins. Nature 345:535-537.

Salinas M, de Weille J, Guillemare E, Lazdunski M, Hugnot JP (1997a) Modes of regulation of Shab $\mathrm{K}^{+}$channel activity by the Kv8.1 subunit. J Biol Chem 272:8774-8780.

Salinas M, Duprat F, Heurteaux C, Hugnot JP, Lazdunski M (1997b) New modulatory $\alpha$-subunits for mammalian Shab $\mathrm{K}^{+}$channels. J Biol Chem 272:24371-24379.

Scannevin RH, Murakoshi H, Rhodes KJ, Trimmer JS (1996) Identification of a cytoplasmic domain important in the polarized expression and clustering of the Kv2.1 K ${ }^{+}$channel. J Cell Biol 135:1619-1632.

Shahidullah M, Hoshi N, Yokoyama S, Kawamura T, Higashida H (1995a) Slow inactivation conserved in heteromultimeric voltagedependent $\mathrm{K}^{+}$channels between Shaker (Kv1) and Shaw (Kv3) subfamilies. FEBS Lett 371:307-310.

Shahidullah M, Hoshi N, Yokoyama S, Higashida H (1995b) Microhet- erogeneity in heteromultimeric assemblies formed by Shaker (Kv1) and Shaw (Kv3) subfamilies of voltage-gated $\mathrm{K}^{+}$channels. Proc R Soc Lond [Biol] 261:309-317.

Shen NV, Pfaffinger PJ (1995) Molecular recognition and assembly sequences involved in the subfamily-specific assembly of voltage-gated $\mathrm{K}^{+}$channel subunit proteins. Neuron 14:625-633.

Shen NV, Chen X, Boyer MM, Pfaffinger PJ (1993) Deletion analysis of $\mathrm{K}^{+}$channel assembly. Neuron 11:67-76.

Sheng M, Liao YJ, Jan YN, Jan LY (1993) Presynaptic A-current based on heteromultimeric $\mathrm{K}^{+}$channels detected in vivo. Nature 365:72-75.

Shi G, Kleinklaus AK, Marrion NV, Trimmer JS (1994) Properties of Kv2.1 K ${ }^{+}$channels expressed in transfected mammalian cells. J Biol Chem 37:23201-23211.

Song W-J, Tkatch T, Baranauskas G, Ichinohe N, Kitai ST, Surmeier DJ (1998) Somatodendritic depolarization-activated potassium currents in rat neostriatal cholinergic interneurons are predominantly of the A type and attributable to coexpression of Kv4.2 and Kv4.1 subunits. J Neurosci 18:3124-3137.

Taglialatela M, Payne JP, Drewe JA, Brown AM (1994) Rescue of lethal subunits into functional $\mathrm{K}^{+}$channels. Biophys J 66:179-190.

Tinker A, Jan YN, Jan LY (1996) Regions responsible for the assembly of inwardly rectifying potassium channels. Cell 87:857-868.

Trimmer JS (1991) Immunological identification and characterization of a delayed-rectifier $\mathrm{K}^{+}$channel polypeptide in rat brain. Proc Natl Acad Sci USA 88:10764-10768.

VanDongen AMJ, Frech GC, Drewe JA, Joho RH, Brown AM (1990) Alteration and restoration of $\mathrm{K}^{+}$channel function by deletions at the $\mathrm{N}$ - and C-termini. Neuron 5:433-443.

Veh RW, Lichtinghagen R, Sewing S, Wunder F, Grumbach IM, Pongs O (1995) Immunohistochemical localization of five members of the Kv1 channel subunits: contrasting subcellular locations and neuron-specific co-localization in rat brain. Eur J Neurosci 7:2189-2205.

Wang H, Kunkel DD, Martin TM, Schwartzkroin PA, Tempel BL (1993) Heteromultimeric $\mathrm{K}^{+}$channels in terminal and juxtaparanodal regions of neurons. Nature 365:75-79.

Weiser M, Vega-Saenz de Miera M, Kentros C, Moreno H, Franzen L, Hillman D, Baker H, Rudy B (1994) Differential expression of Shawrelated $\mathrm{K}^{+}$channels in the rat central nervous system. J Neurosci 14:949-972

Xu J, Yu W, Jan YN, Jan LY, Li M (1995) Assembly of voltage-gated potassium channels. J Biol Chem 270:24761-24768.

Young Y, Yan Y-Y, Sigworth FJ (1997) How does the W434F mutation block current in Shaker potassium channels? J Gen Physiol 109:779-789. 